\title{
Product Integration-Collocation Methods for Noncompact Integral Operator Equations*
}

\author{
By G. A. Chandler and I. G. Graham
}

\begin{abstract}
We discuss the numerical solution of a class of second-kind integral equations in which the integral operator is not compact. Such equations arise, for example, when boundary integral methods are applied to potential problems in a two-dimensional domain with corners in the boundary. We are able to prove the optimal orders of convergence for the usual collocation and product integration methods on graded meshes, provided some simple modifications are made to the underlying basis functions. These are sufficient to ensure stability, but do not damage the rate of convergence. Numerical experiments show that such modifications are necessary in certain circumstances.
\end{abstract}

1. Introduction. This paper considers the numerical solution of the second-kind integral equation

$$
u(s)-(\mathscr{K} u)(s)=f(s), \quad s \in[0,1],
$$

where $u$ is the unknown solution, $f$ is given and $\mathscr{K}$ is the integral operator

$$
(\mathscr{K} u)(s)=\int_{0}^{1} K\left(\frac{s}{\sigma}\right) u(\sigma) \frac{d \sigma}{\sigma}, \quad s \in(0,1]
$$

for some given kernel function $K$. Such equations arise in a variety of contexts, most commonly when boundary integral methods are used on domains with corners. The difficulty is that $\mathscr{K}$ is not compact, and the standard stability proofs for numerical methods ([1]) do not apply.

We consider the practically important case in which the approximate solution is a piecewise polynomial, $u_{n}$. If $u_{n}$ is calculated by a Galerkin method, fairly complete stability results are known ([8], [9], [11], [12], [19]). However, Galerkin methods are expensive to implement and collocation methods are used more often in practice, for instance in the boundary element method of the engineering literature (see [5]). The numerical analysis of these methods is incomplete. The difficulty is to prove the stability result that the system of linear equations defining $u_{n}$ is nonsingular. Previous work has established stability in special cases ([2], [20], [24], [30]), and numerical experiments have suggested that collocation methods converge at the rates

Received November 5, 1985; revised August 13, 1986 and April 1, 1987.

1980 Mathematics Subject Classification (1985 Revision). Primary 65R20, 45B05.

Key words and phrases. Second-kind integral equations, product integration, boundary integral equations, collocation.

* This work was supported by a visiting fellowship at the Centre for Mathematical Analysis, Australian National University, Canberra. 
which could be proved if $\mathscr{K}$ happened to be compact ([2], [20]). We show here that this is not always true, and Section 4 contains an example in which collocation diverges.

However, we can prove a general stability result if the collocation method is slightly modified. High-order piecewise polynomial approximations are replaced by piecewise constants on a number of the intervals closest to $s=0$ (the point at which $\mathscr{K}$ is badly behaved). While ensuring the stability of the method, the rate of convergence is not damaged.

2. Preliminary Analysis. To describe some analytic results for (1.1), we introduce $\mathscr{C}$, the Banach space of uniformly continuous functions on $[0,1]$, equipped with the uniform norm $\|\cdot\|$. If $\mathscr{L}$ is a bounded linear operator on $\mathscr{C},\|\mathscr{L}\|$ will denote the norm of $\mathscr{L}$.

For $\alpha>0$ and integer $k \geqslant 0$ define $\mathscr{C}_{\alpha}^{k}$ to be the subspace of $\mathscr{C}$ consisting of functions for which the seminorm

$$
|v|_{k, \alpha}:= \begin{cases}\sup _{0<\sigma \leqslant 1}\left|\sigma^{k-\alpha} D^{k} v(\sigma)\right|, & k>\alpha, \\ \left\|D^{k} v\right\|, & k \leqslant \alpha,\end{cases}
$$

is finite. Then $\mathscr{C}_{\alpha}^{k}$ is a Banach space under the norm

$$
\|v\|_{k, \alpha}:=\max \left\{|v|_{l, \alpha}: 0 \leqslant l \leqslant k\right\} .
$$

Our assumptions on $K$ are

A1: for all integers $k \geqslant 0$

$$
B_{k}:=\int_{0}^{\infty} \sigma^{k}\left|D^{k} K(\sigma)\right| \frac{d \sigma}{\sigma}<\infty,
$$

A2:

$$
B_{0}=\int_{0}^{\infty}|K(\sigma)| \frac{d \sigma}{\sigma}<1,
$$

A3: there exists $\alpha^{*}>0$ such that for all integers $k \geqslant 0, u \in \mathscr{C}_{\alpha^{*}}^{k}$.

Under A2, $\mathscr{K}$ is a contraction on $\mathscr{C}([2])$, and by the Banach lemma, (1.1) has a unique solution $u \in \mathscr{C}$. The more stringent assumption $\mathrm{A} 1$ is needed to prove that the numerical methods of Section 3 converge at high rates. In practice this is not an extra restriction, for if $K$ is a rational function, $B_{0}<\infty$ implies $B_{k}<\infty$ for all $k>0$.

With the change of variables $s=e^{-t}, \sigma=e^{-\tau}$, (1.1) becomes the Wiener-Hopf equation

$$
u\left(e^{-t}\right)-\int_{0}^{\infty} K\left(e^{-(t-\tau)}\right) u\left(e^{-\tau}\right) d \tau=f\left(e^{-t}\right), \quad t \geqslant 0 .
$$

Known results about (2.1) can then be easily transformed back to results about (1.1). Recall that the Mellin transform is defined by

$$
\hat{v}(\omega)=\int_{0}^{\infty} \sigma^{i \omega} v(\sigma) \frac{d \sigma}{\sigma}
$$

(equivalently, $\hat{v}$ is the Fourier transform of $t \mapsto v\left(e^{-t}\right)$ ). It follows from Krein [18, Theorem I] that the spectrum of $\mathscr{K}$ contains the nondiscrete set $\{\hat{K}(\omega): \omega \in \mathbf{R}\}$. Hence $\mathscr{K}$ cannot be compact. 
A3 is more difficult to verify. However, using Mellin transform techniques it can be shown (see [11], [12], [20] for instance) that A3 is true for all smooth right-hand sides provided:

for some $\varepsilon>0,1 /(1-\hat{K}(\omega))$ is analytic in the strip

$\left\{\omega \in \mathbf{C}: 0 \leqslant \operatorname{Im}(\omega)<\alpha^{*}+\varepsilon\right\}$ except for simple poles in the set $\{i k: k \in \mathbf{N}\}$.

(See [8] for a simpler approach not using Mellin transforms.) Numerically, the important point is that even if $f$ is smooth, singularities appear in the solution at $s=0$. If $(2.2)$ is satisfied for $\alpha^{*} \in(0, \beta)$ and $1 /(1-\hat{K}(\omega))$ has a single pole at $\omega=i \beta(\beta \notin \mathbf{N})$, then

$$
u(s)=a s^{\beta}+v(s)
$$

where $a$ is an unknown constant and $v$ is a smoother function than $s^{\beta}$ (more precisely, $v \in \mathscr{C}_{\beta^{\prime}}^{k}$ for some $\beta^{\prime}>\beta$ ). Numerical techniques must be modified to cope with this behavior.

We give two examples of these problems arising in applications.

Example 1 (Potential Theory). Let $\Omega \subset \mathbf{R}^{2}$ be a simply connected domain with boundary $\Gamma$. Suppose $U: \Omega \rightarrow \mathbf{R}$ is the solution to the interior Dirichlet problem

$$
(\Delta U)(x)=0,\left.\quad U\right|_{\Gamma}=g \text {, }
$$

where $g: \Gamma \rightarrow \mathbf{R}$ is given and $\Delta=\nabla \cdot \nabla$ is the Laplacian. Define

$$
G^{\prime}(x, \xi)=\frac{\partial}{\partial \nu(\xi)} \frac{1}{2 \pi} \ln \frac{1}{|x-\xi|}, \quad x \in \mathbf{R}^{2}, \xi \in \Gamma,
$$

the derivative of the fundamental solution with respect to the outward normal at $\xi$. Then it is known [16] that

$$
U(x)=\left(\mathscr{V}_{D} u\right)(x):=\int_{\Gamma} G^{\prime}(x, \cdot) u,
$$

where the double layer source $u$ is the solution of the boundary integral equation

$$
u(x)-2\left(\mathscr{V}_{D} u\right)(x)=-2 g(x), \quad x \in \Gamma .
$$

If $\Gamma$ is smooth, the integral operator in (2.3) is compact and the standard numerical analysis applies. But suppose $\Gamma$ has corners. That is, $\Gamma$ may be divided into smooth segments $\Gamma_{1}, \ldots, \Gamma_{M}$ with $\Gamma_{l-1}$ and $\Gamma_{l}$ joining at a corner $x_{l}$ with an exterior angle $\chi_{1} \pi, 0<\chi_{1}<2$. Then for all $x \in \Gamma_{1-1}$ with $x$ closer to the $x_{1}$ than $x_{1-1}$,

$$
2\left(\mathscr{V}_{D} u\right)(x)=\int_{\Gamma_{l}} K_{\chi_{l}}\left(\frac{\left|x-x_{l}\right|}{\left|\xi-x_{l}\right|}\right) \frac{u(\xi) d \xi}{\left|\xi-x_{l}\right|}+\left(\mathscr{R}_{l} u\right)(x),
$$

where $\mathscr{R}$, is a compact operator and

$$
K_{\chi}(\sigma):=\frac{\sin \chi \pi}{\pi} \frac{\sigma}{1+\sigma^{2}-2 \sigma \cos \chi \pi} .
$$

Hence (2.3) may be rewritten as a system involving equations of the form

$$
u(s)-\int_{0}^{1} K_{\chi}\left(\frac{s}{\sigma}\right) u(\sigma) \frac{d \sigma}{\sigma}=f(s), \quad \chi \in\left\{\chi_{l}, 2-\chi_{l}\right\}
$$

(see [2], [8], [20]). The treatment of the system is standard, once (2.4) is understood. 
In (2.4), $\hat{K}_{\chi}(\omega)=\sinh ((1-\chi) \pi \omega) / \sinh (\pi \omega)$, and A3 holds for $\alpha^{*}<\beta$, with $\beta:=\min \left\{(2-\chi)^{-1}, \chi^{-1}\right\}$ (by (2.2)). A more detailed analysis ([12]) shows the solution (2.4) contains a singularity of the form $s^{\beta}$. This corresponds to results in [17] about the singularities found in $U$.

A potentially useful technique is to reformulate (2.4) as an equation with the smoother unknown $v(s)=(u(s)-u(0)) / s^{\beta}([13])$. However, the resulting integral equation (of the form (1.1)) will not have a unique solution (as 1 belongs to the spectrum of the integral operator).

Example 2 (Plane Strain Elasticity). A second class of examples occurs when transform techniques [26] are applied to crack problems in elasticity. For example, [25] and [28] consider the case of an elastic material in the half plane $\left\{\left(x_{1}, x_{2}\right)\right.$ : $\left.x_{1} \geqslant 0\right\}$ with a crack $\{(x, 0): 0 \leqslant x \leqslant 1\}$ being opened by an internal pressure distribution. This is reduced to an equation of the form (1.1) with kernel function

$$
K(\sigma)=\frac{16}{\pi^{2}} \frac{\sigma^{2}}{\left(\sigma^{2}-1\right)^{2}}\left\{\frac{\sigma^{2}+1}{\sigma^{2}-1} \ln \sigma-1\right\} .
$$

A1 and A2 may be verified and as $\hat{K}(\omega)=\omega^{2} / \sinh ^{2}(\pi \omega / 2)$, A3 is satisfied for $\alpha^{*} \leqslant 2.739 \ldots$. This reformulation is useful as the stress intensity factor and crack energy are simply found from $u$ ([15], [25], [28]). A similar equation arises in [27].

We conclude this section by stating a technical result which follows from A1. It introduces the useful notation

$$
K_{s}(\sigma):=K\left(\frac{s}{\sigma}\right) \frac{1}{\sigma}, \quad \sigma \in(0,1] .
$$

The proof follows by calculating derivatives explicitly (see [10]).

LEMMA 1. (a) For all $k \geqslant 0$, there is a constant $C_{k}$ independent of $s$ and $\sigma$ such that

$$
\left|\sigma^{k+1} D^{k} K_{s}(\sigma)\right| \leqslant C_{k}, \quad \sigma \in(0,1] .
$$

(b) For all $v \in \mathscr{C}, \mathscr{K} v$ has derivatives of all orders on $(0,1]$ and, for all $k \geqslant 0$,

$$
\left|s^{k}\left(D^{k} \mathscr{K} v\right)(s)\right| \leqslant B_{k}\|v\|, \quad s \in(0,1] .
$$

3. Numerical Solutions. We consider the solution of the second-kind equation (1.1) by methods based on piecewise polynomials. Thus suppose there is a sequence of meshes $\left\{x_{i}^{(n)} ; 0 \leqslant i \leqslant n\right\}$ with $0=x_{0}^{(n)}<x_{1}^{(n)}<\cdots<x_{n}^{(n)}=1$. For convenience write $x_{i}$ for $x_{i}^{(n)}$ where possible, and let $I_{i}=\left(x_{i-1}, x_{i}\right)$ and $h_{i}=x_{i}-x_{i-1}$. For any function $v:[0,1] \rightarrow \mathbf{R}, v_{i}$ denotes $\left.v\right|_{I_{i}}$. Let $S_{n}$ denote the set of piecewise polynomials of order $r$ (i.e., degree $r-1$ ) on this grid. That is, $\phi \in S_{n}$ if and only if $\phi_{i} \in \mathbf{P}_{r}$ $\left(\mathbf{P}_{r}\right.$ denotes the polynomials of order $r$ ). There are no continuity restrictions imposed on $S_{n}$ and the discontinuity $\phi_{i}\left(x_{i}\right) \neq \phi_{i+1}\left(x_{i}\right)$ is permitted. To utilize the full potential of piecewise polynomials, it is necessary to use graded meshes near singularities ([3], [4], [6], [7], [14], [21], [22]).

Definition. For an integer $k \geqslant 1$ and $\alpha \in(0, k]$ the meshes $\left\{x_{i}^{(n)}\right\}$ are defined to be $(k, \alpha)$-graded if there is a constant $\gamma$ independent of $n$ so that

$$
h_{i}<\frac{\gamma}{n} x_{i-}^{1-\alpha / k}, \quad i=1,2, \ldots, n .
$$


(Here we define $i-:=\max \{i-1,1\}$.) Condition (3.1) with $k=r$ is exactly the condition needed on the mesh so that $S_{n}$ approximates $s^{\alpha}$ to within $O\left(1 / n^{r}\right)([4$, Chapter XII]). The simplest example of such a mesh is ([21])

$$
x_{i}^{(n)}=\left(\frac{i}{n}\right)^{q}, \quad i=0,1, \ldots, n .
$$

These meshes are $(k, \alpha)$-graded if $q \geqslant k / \alpha$. Condition (3.1) applies to more general meshes that may be generated adaptively by successive local refinement ([7]). If $\alpha>k,(k, \alpha)$-graded is defined to mean $(k, k)$-graded.

To state the collocation method, introduce the points $\left\{\xi_{j}: 1 \leqslant j \leqslant r\right\}$ with $0 \leqslant \xi_{1}<\xi_{2}<\cdots<\xi_{r} \leqslant 1$, and define the collocation points

$$
x_{i j}=x_{i}+\xi_{j} h_{i}, \quad 1 \leqslant i \leqslant n, 1 \leqslant j \leqslant r .
$$

For any function $v$, continuous at $\left\{x_{i j}\right\}$, define $Q_{n} v \in S_{n}$ by

$$
\left(Q_{n} v\right)_{i}\left(x_{i j}\right)=v_{i}\left(x_{i j}\right), \quad 1 \leqslant i \leqslant n, 1 \leqslant j \leqslant r .
$$

The collocation solution to the integral equation is the piecewise polynomial $u_{n} \in S_{n}$ satisfying

$$
\left(I-Q_{n} \mathscr{K}\right) u_{n}=Q_{n} f .
$$

The product integration solution is defined by

$$
\left(I-\mathscr{K} Q_{n}\right) u_{n}^{*}=f
$$

or equivalently

$$
u_{n}^{*}=f+\mathscr{K} u_{n}
$$

(see [1], [23]). When a basis is selected for $S_{n}$, (3.3) becomes a system of linear equations of $\operatorname{dimension} \operatorname{dim}\left(S_{n}\right)$. As $Q_{n} u_{n}^{*}=u_{n}, u_{n}^{*}\left(x_{i j}\right)=u_{n}\left(x_{i j}\right)$, and once the collocation equations have been solved, $u_{n}^{*}$ is available at the collocation points. Equation (3.5) can then be used to calculate $u_{n}^{*}$ elsewhere if required.

The rate of convergence of $u_{n}^{*}$ depends on the careful positioning of the $\xi_{i}$. For any $v:[0,1] \rightarrow \mathbf{R}$ let $Q v$ denote the polynomial of order $r$ interpolating $v$ at $\xi_{1}, \ldots, \xi_{r}$. We assume that for some $r^{\prime} \geqslant 0$, and for all $\phi \in \mathbf{P}_{r+r^{\prime}}$,

$$
\int_{0}^{1} \phi=\int_{0}^{1} Q \phi \text {. }
$$

This is equivalent to requiring for all $\psi \in \mathbf{P}_{r^{\prime}}$

$$
\int_{0}^{1}\left(\prod_{i=1}^{r}\left(\xi-\xi_{i}\right)\right) \psi(\xi) d \xi=0
$$

or alternatively, for all integers $\nu \in\left[0, r^{\prime}\right]$ and for all $\phi \in \mathbf{P}_{r+r^{\prime}-\nu}$ and $\psi \in \mathbf{P}_{\nu+1}$

$$
\int_{0}^{1} \phi \psi=\int_{0}^{1}(Q \phi) \psi \text {. }
$$

Equation (3.7) shows that the maximum value of $r^{\prime}$ is $r$, in which case $\left\{\xi_{i}\right\}$ must be the $r$ Gauss points on $[0,1]$. Other choices are also useful; if $\left(\xi_{1}, \xi_{2}, \xi_{3}\right)=\left(0, \frac{1}{2}, 1\right)$ then $r^{\prime}=1$ and $u_{n}^{*}$ is the product integration Simpson's rule approximation.

It is straightforward to use (3.3) and (1.1) to show ([1])

$$
\left(I-Q_{n} \mathscr{K}\right)\left(u_{n}-u\right)=-Q_{n}^{\prime} u
$$


$\left(Q_{n}^{\prime}:=I-Q_{n}\right)$. Hence, if the collocation equations are nonsingular, $u_{n}$ is uniquely defined and

$$
\left\|u_{n}-u\right\| \leqslant\left\|\left(I-Q_{n} \mathscr{K}\right)^{-1}\right\|\left\|Q_{n}^{\prime} u\right\|
$$

Further, (3.4) and (3.5) give

$$
u_{n}^{*}-u=-\left(I+\mathscr{K}\left(I-Q_{n} \mathscr{K}\right)^{-1} Q_{n}\right)\left(\mathscr{K} Q_{n}^{\prime} u\right),
$$

and therefore, as $\left\|Q_{n}\right\|$ is bounded,

$$
\left\|u_{n}^{*}-u\right\| \leqslant C\left\|\left(I-Q_{n} \mathscr{K}\right)^{-1}\right\|\left\|\mathscr{K} Q_{n}^{\prime} u\right\| .
$$

(Here and elsewhere $C$ is used to denote a generic constant independent of $n$ and the solution $u$.) Once we have proved the stability result

$$
\left\|\left(I-Q_{n} \mathscr{K}\right)^{-1}\right\| \leqslant C,
$$

the rate of convergence of $u_{n}$ and $u_{n}^{*}$ is governed by the discretization errors $\left\|Q_{n}^{\prime} u\right\|$ and $\left\|\mathscr{K} Q_{n}^{\prime} u\right\|$, respectively. But the discretization errors are relatively easy to bound. As $u \in \mathscr{C}_{\alpha^{*}}^{r}$ it follows from [21] that

$$
\left\|Q_{n}^{\prime} u\right\|=O\left(1 / n^{r}\right)
$$

provided the mesh is $\left(r, \alpha^{*}\right)$-graded. From [20], for example, we also have

$$
\left\|\mathscr{K} Q_{n}^{\prime} u\right\|=O\left(1 / n^{r+r^{\prime}}\right)
$$

for meshes (3.2) which are $\left(r+r^{\prime}, \alpha\right)$-graded for $\alpha<\alpha^{*}$. These are the same optimal rates of convergence observed when collocation is applied to an equation with a smooth kernel and solution.

However, the accompanying stability result (3.11) has only been proved in special cases. When $r=0$ then $\left\|Q_{n}\right\|=1$, and stability follows from $\left\|Q_{n} \mathscr{K}\right\| \leqslant\left\|Q_{n}\right\|\|\mathscr{K}\|$ $=\|\mathscr{K}\|<1$ and the Banach lemma. Similarly, if $r=1$ and the product trapazoidal rule is used (i.e., $\xi_{0}=0, \xi_{1}=1$ ), then $\left\|Q_{n}\right\|=1$ (see [2]). But no results are known for methods which give rates of convergence higher than 2. (The stability of the product 2-point Gauss rule is proved only for a uniform mesh in [2], and this gives only $O\left(1 / n^{\beta}\right)$ convergence if $u$ contains the typical singularity $s^{\beta}$. See [20].) Moreover, the counterexample of Section 4 shows that stability does not hold in general unless some (as yet unknown) conditions are placed on $K$ and $\left\{\xi_{1}, \ldots, \xi_{r}\right\}$.

The source of the difficulty is that the kernel of $\mathscr{K}, K(s / \sigma) 1 / \sigma$ becomes unbounded as $s$ and $\sigma$ approach 0 . Thus, if $\phi$ is one of the basis functions of $S_{n}$ with support in one of the subintervals closest to 0 , the coefficient $(\mathscr{K} \phi)\left(x_{i j}\right)$ in the collocation equations is relatively large if $x_{i j}$ is also close to 0 . These large entries cause "spurious" eigenvalues in the approximate operator $Q_{n} \mathscr{K}$, that is, eigenvalues which are outside the spectrum of the true operator $\mathscr{K}$. As $n$ increases, these eigenvalues converge to a limit point which is still outside the spectrum of $\mathscr{K}$. (An observation first made in [20]; see also [10].) If this limit happens to be 1 , then $I-Q_{n} \mathscr{K}$ becomes increasingly singular as $n \rightarrow \infty$ and the collocation solution diverges. This can be corrected by modifying the intervals closest to 0 to remove the large coefficients in the collocation equations. If this is done for only a small proportion of the subintervals of the mesh, the rate of convergence of the discretization error is undamaged. 
Definition. Consider the piecewise polynomials $S_{n}$ on the meshes $\left\{x_{i}^{(n)}\right\}$. A modification is determined by a sequence $i(n)$, with $0 \leqslant i(n)<n$. The modification is $(k, \alpha)$-acceptable if there is a constant $\bar{\gamma}$ independent of $n$ such that

$$
\left(x_{i(n)}^{(n)}\right)^{\alpha / k} \leqslant \bar{\gamma} / n .
$$

The modified basis functions are defined by

$$
\bar{S}_{n}:=\left\{\phi \in S_{n}: i \leqslant i(n) \Rightarrow \phi_{i} \in \mathbf{P}_{1}\right\} .
$$

The modified basis functions are those functions in $S_{n}$ which are piecewise constants on $\left[0, x_{l(n)}^{(n)}\right]$. If the mesh is $(k, \alpha)$-graded, the modification is $(k, \alpha)$-acceptable if the coarser mesh $\left\{x_{i}^{(n)}: i=0, i \geqslant i(n)\right\}$ is still $(k, \alpha)$-graded. For the meshes (3.2) the modification is $(k, \alpha)$-acceptable provided

$$
i(n) \leqslant \bar{\gamma}^{\prime} n^{1-k / \alpha q}
$$

for some constant $\bar{\gamma}^{\prime}$. The following technical lemma is needed in the proof of Theorem 4.

Lemma 2. Suppose the mesh $\left\{x_{i}^{(n)}\right\}$ is $(k, \alpha)$-graded. Then for all $\varepsilon>0$ and $n$ sufficiently large there exists $i(\varepsilon, n) \leqslant n$ such that

$$
\left(x_{i(\varepsilon, n)}^{(n)}\right)^{\alpha / k} \leqslant\left(1+\frac{1}{\varepsilon}\right) \frac{\gamma}{n},
$$

and

$$
i>i(\varepsilon, n) \Rightarrow h_{i} / x_{i-} \leqslant \varepsilon .
$$

Proof. Define

$$
i(\varepsilon, n)=\min \left\{\iota \geqslant 0: i>\iota \Rightarrow h_{i} / x_{i-} \leqslant \varepsilon\right\} .
$$

(As $h_{n} \leqslant O(1 / n)$, the set is nonempty for $n$ sufficiently large.) Clearly (3.15) is satisfied, and (3.14) is satisfied if $i(\varepsilon, n) \leqslant 1$. When $i(\varepsilon, n)>1$, the definition of $i(\varepsilon, n)$ gives $h_{i(\varepsilon, n)} / x_{i(\varepsilon, n)-}>\varepsilon$ and hence

$$
x_{i(\varepsilon, n)}=x_{i(\varepsilon, n)-}+h_{i(\varepsilon, n)} \leqslant h_{i(\varepsilon, n)}(1+1 / \varepsilon) .
$$

Combined with the mesh grading, this gives

$$
x_{i(\varepsilon, n)} \leqslant \frac{\gamma}{n} x_{i(\varepsilon, n)-}^{1-\alpha / k}(1+1 / \varepsilon) \leqslant \frac{\gamma}{n} x_{i(\varepsilon, n)}^{1-\alpha / k}(1+1 / \varepsilon) \leqslant\left(1+\frac{1}{\varepsilon}\right) \frac{\gamma}{n}
$$

(as $\alpha \leqslant k$ ).

A modified collocation projection $\bar{Q}_{n}$ onto $\bar{S}_{n}$ is defined by

$$
\left(\bar{Q}_{n} v\right)_{i}= \begin{cases}\left(Q_{n} v\right)_{i}, & i>i(n), \\ v\left(x_{i-1 / 2}\right), & i \leqslant i(n)\end{cases}
$$

(where $x_{i-1 / 2}:=\frac{1}{2}\left(x_{i-1}+x_{i}\right)$ ). The approximation properties of $\bar{Q}_{n}$ are described in Lemma 3. The proof can be obtained by imitating [21] for instance. See also [10].

LEMMA 3. Let $\overline{S_{n}}$ be a modification of $S_{n}$.

(a) If $v$ has $r$ continuous derivatives on $\left(x_{i-1}, x_{i}\right)$ for $i>i(n)$, then

$$
\left\|\left(\bar{Q}_{n} v-v\right)_{i}\right\| \leqslant C h_{i}^{r}\left\|\left(D^{r} v\right)_{i}\right\| .
$$

(b) If $v \in \mathscr{C}_{\alpha}^{1}$ for some $\alpha \leqslant 1$, then for all $i \leqslant i(n)$

$$
\left\|\left(\bar{Q}_{n} v-v\right)_{i}\right\| \leqslant C h_{i}^{\alpha}\|v\|_{1, \alpha} .
$$


(c) If the mesh underlying $S_{n}$ is $(r, \alpha)$-graded and $\bar{S}_{n}$ is an $(r, \alpha)$-acceptable modification for $\alpha \leqslant 1$, then for all $v \in \mathscr{C}_{\alpha}^{r},\left\|Q_{n} v-v\right\| \leqslant C\|v\|_{r, \alpha} / n^{r}$.

The modified projection $\bar{Q}_{n}$ may be used to define the modified collocation solution $\bar{u}_{n} \in \bar{S}_{n}$ by

$$
\bar{u}_{n}-\bar{Q}_{n} \mathscr{K} \bar{u}_{n}=\bar{Q}_{n} f
$$

and the modified product integration solution $\bar{u}_{n}^{*}$ by

$$
\bar{u}_{n}^{*}-\mathscr{K} Q_{n} \bar{u}_{n}^{*}=f
$$

or

$$
\bar{u}_{n}^{*}=f+\mathscr{K} \bar{u}_{n} .
$$

Again, (3.16) is a system of linear equations which is closely related to the unmodified collocation equations. Suppose the basis for the unmodified $S_{n}$ includes the characteristic functions for each interval. Then the matrix for (3.16) is obtained by eliminating $i(n)(r-1)$ rows and columns from the unmodified matrix. Thus the modified method is as easy to implement as the original collocation method.

Our stability and consistency results are given in Theorems 4 and 5.

THEOREM 4. Suppose the mesh underlying $S_{n}$ is $(k, \alpha)$-graded and that $\delta$ satisfies $\|\mathscr{K}\|<\delta<1$. Then there exists $a(k, \alpha)$-acceptable modification $i_{\delta}(n)$ such that

$$
\left\|\bar{Q}_{n} \mathscr{K}\right\| \leqslant \delta
$$

for all $n$ sufficiently large. Moreover, (3.18) is satisfied for any other modification $i(n)$ for which $i(n) \geqslant i_{\delta}(n)$ for all $n$.

Proof. To prove (3.18) it must be shown that $i_{\delta}(n)$ can be selected so that for all $v \in \mathscr{C}$

$$
\left\|\left(\bar{Q}_{n} \mathscr{K} v\right)_{i}\right\| \leqslant \delta\|v\|
$$

for all $i$. For any $i \leqslant i_{\delta}(n),(3.19)$ is true, as A2 shows for $s \in I_{i}$ :

$$
\left|\left(\bar{Q}_{n} \mathscr{K} v\right)(s)\right|=\left|\mathscr{K}_{v}\left(x_{i-1 / 2}\right)\right| \leqslant\|\mathscr{K}\|\|v\| .
$$

Suppose $i>i_{\delta}(n)$. Then the triangle inequality, Lemma 3(a) and Lemma 1(b) give

$$
\begin{aligned}
\left\|\left(\bar{Q}_{n} \mathscr{K} v\right)_{i}\right\| & \leqslant\left\|(\mathscr{K} v)_{i}\right\|+\left\|\left(\left(I-\bar{Q}_{n}\right) \mathscr{K}_{v_{i}}\right)\right\| \\
& \leqslant\|\mathscr{K}\|\|v\|+C h_{i}^{r}\left\|\left(D^{r} \mathscr{K} v\right)_{i}\right\| \\
& \leqslant\|\mathscr{K}\|\|v\|+C_{1}\left(h_{i} / x_{i-}\right)^{r}\|v\|,
\end{aligned}
$$

where the constant $C_{1}$ is independent of $n, i$ and $v$. But now choose $i_{\delta}(n)=$ $\max \{1, i(\varepsilon, n)\}$, where $i(\varepsilon, n)$ is the integer given by Lemma 2 when $\varepsilon=$ $\left((\delta-\|\mathscr{K}\|) / C_{1}\right)^{1 / r}$. By $(3.14)$ this is $(k, \alpha)$-acceptable and by $(3.15)$

$$
\left(h_{i} / x_{i-}\right)^{r} \leqslant(\delta-\|\mathscr{K}\|) / C_{1} .
$$

(3.21) and (3.20) give (3.19) as required. Clearly this proof holds if $i(n) \geqslant i_{\delta}(n)$.

TheOREM 5. Suppose $v \in \mathscr{C}_{\alpha}^{r+r^{\prime}}$ for $\alpha \leqslant 1$ and the mesh underlying $S_{n}$ is $\left(r+r^{\prime}, \beta\right)$-graded for $\beta<\alpha$. Then for any $\left(r+r^{\prime}, \alpha\right)$-acceptable modification,

$$
\left\|\mathscr{K} \bar{Q}_{n}^{\prime} v\right\| \leqslant \frac{C}{n^{r+r^{\prime}}}\|v\|_{r+r^{\prime}, \alpha} .
$$


Proof. It is convenient to write $\rho=r+r^{\prime}$ and $i 1=\max \{1, i(n)\}$, where $i(n)$ is the modification used in defining $\bar{Q}_{n}$. Let $(\cdot, \cdot)_{i}$ be defined by

$$
\left(v_{1}, v_{2}\right)_{i}=\int_{I_{i}} v_{1} v_{2} \text {. }
$$

Then

$$
\left(\mathscr{K} \bar{Q}_{n}^{\prime} v\right)(s)=\mathscr{K}\left(\bar{Q}_{n}^{\prime} v\right)_{i 1}(s)+\sum_{i>i 1}\left(K_{s}, Q_{n}^{\prime} v\right)_{i},
$$

where $\left(\bar{Q}_{n}^{\prime} v\right)_{i 1}=\left.\bar{Q}_{n}^{\prime} v\right|_{\left[0, x_{i 1}\right]}$. We need to estimate the two terms of (3.23).

For $i>i 1$ let $\phi_{\nu}, \nu=r, r+1, \ldots, \rho$, denote the Taylor polynomial of order $\nu$ for $v$ about $x_{i}$. Then

$$
\left(K_{s}, Q_{n}^{\prime} v\right)_{i}=\left(K_{s}, Q_{n}^{\prime}\left(v-\phi_{\rho}\right)\right)_{i}+\sum_{\nu=r+1}^{\rho}\left(K_{s}, Q_{n}^{\prime}\left(\phi_{\nu}-\phi_{\nu-1}\right)\right)_{i} .
$$

But it follows from the properties of the Taylor polynomial that

$$
\left\|\left(v-\phi_{\rho}\right)_{i}\right\| \leqslant C h_{i}^{\rho}\left\|\left(D^{\rho} v\right)_{i}\right\|
$$

and

$$
\left\|\left(\phi_{\nu}-\phi_{\nu-1}\right)_{i}\right\| \leqslant C h_{i}^{\nu-1}\left\|\left(D^{\nu-1} v\right)_{i}\right\| .
$$

We can also approximate $K_{s}$ by a polynomial $\psi_{\nu}$ of order $\rho+1-\nu$ so that

$$
\left\|\left(K_{s}-\psi_{\nu}\right)_{i}\right\| \leqslant C h_{i}^{\rho+1-\nu}\left\|D^{\rho+1-\nu} K_{s}\right\| \leqslant C\left(h_{i} / x_{i-}\right)^{\rho+1-\nu} x_{i-}^{-1}
$$

(using Lemma 1(a)). Hence, using (3.8) on $I_{i}$, and (3.25)-(3.27),

$$
\begin{aligned}
\left|\left(K_{s}, Q_{n}^{\prime}\left(\phi_{\nu}-\phi_{\nu-1}\right)\right)_{i}\right| & =\left|\left(K_{s}-\psi_{\nu}, Q_{n}^{\prime}\left(\phi_{\nu}-\phi_{\nu-1}\right)\right)_{i}\right| \\
& \leqslant h_{i}\left\|\left(K_{s}-\psi_{\nu}\right)_{i}\right\|\left\|\left(\phi_{\nu}-\phi_{\nu-1}\right)_{i}\right\| \\
& \leqslant C\left(h_{i}^{\rho+1} / x_{i-}^{\rho-\nu+2}\right)\left\|\left(D^{\nu-1} v\right)_{i}\right\| \\
& \leqslant C\left(h_{i}^{\rho+1} x_{i-}^{\beta-\rho-1}\right)\|v\|_{\rho, \beta} .
\end{aligned}
$$

Further from (3.25) and Lemma 1(a),

$$
\left|\left(K_{s}, Q_{n}^{\prime}\left(v-\phi_{\rho}\right)\right)_{i}\right| \leqslant C h_{i}^{\rho+1} x_{i-}^{\beta-\rho-1}\|v\|_{\rho, \beta} .
$$

Therefore, combining (3.28) and (3.29) gives bounds for each term on the right of (3.24). Summing over $i>i 1$ and using the mesh grading gives

$$
\begin{aligned}
\left|\sum_{i>i 1}\left(K_{s}, Q_{n}^{\prime} v\right)_{i}\right| & \leqslant C\left(\sum_{i>i 1} h_{i}^{\rho+1} x_{i-}^{\beta-\rho-1}\right)\|v\|_{\rho, \beta} \\
& \leqslant C\left(\sum_{i>i 1}\left(h_{i} x_{i-}^{\beta-\alpha-1}\right)\left(h_{i}^{\rho} x_{i-}^{\alpha-\rho}\right)\right)\|v\|_{\rho, \beta} \\
& \leqslant \frac{C}{n^{\rho}}\left(\sum_{i>i 1} h_{i} x_{i-}^{\beta-\alpha-1}\right)\|v\|_{\rho, \beta} \leqslant \frac{C}{n^{\rho}}\|v\|_{\rho, \beta},
\end{aligned}
$$

where the last inequality follows because the last sum is a Riemann sum for the finite integral

$$
\int_{x_{i 1}}^{1} \sigma^{\beta-\alpha-1} d \sigma
$$


To bound the first term of (3.23), use Lemma 3(b) to give

$$
\left\|\mathscr{K}\left(\bar{Q}_{n}^{\prime} v\right)_{i 1}\right\| \leqslant\|\mathscr{K}\|\left\|\left(\bar{Q}_{n}^{\prime} v\right)_{i 1}\right\| \leqslant C x_{i 1}^{\alpha}\|v\|_{\rho, \alpha .}
$$

Hence the acceptability of the modification shows

$$
\left\|\mathscr{K}\left(Q_{n}^{\prime} v\right)_{i 1}\right\| \leqslant \frac{C}{n^{\rho}}\|v\|_{\rho, \alpha} .
$$

Finally, as $\|v\|_{\rho, \beta} \leqslant\|v\|_{\rho, \alpha}$, (3.30) and (3.31) give the required bounds for the right-hand side of (3.23).

Our stability and consistency results are then combined to give convergence.

THEOREM 6. Suppose the mesh underlying $S_{n}$ is $\left(r+r^{\prime}, \alpha\right)$-graded for $\alpha$ satisfying $\alpha \leqslant 1$ and $\alpha<\alpha^{*}$. Then there exists a modification such that for all $n$ sufficiently large, $\bar{u}_{n}$ and $\bar{u}_{n}^{*}$ are uniquely defined and

$$
\left\|\bar{u}_{n}^{*}-u\right\| \leqslant \frac{C}{n^{r+r^{\prime}}}\|u\|_{r+r^{\prime}, \alpha^{*}}
$$

Proof. As the mesh is $\left(r+r^{\prime}, \alpha\right)$-graded, it is $\left(r+r^{\prime}, \bar{\alpha}^{*}\right)$-graded for $\bar{\alpha}^{*}:=\min \left\{\alpha^{*}, 1\right\}$. Theorem 4 shows that there is an $\left(r+r^{\prime}, \bar{\alpha}^{*}\right)$-acceptable modification so that (3.18) holds for some $\delta \in(\|K\|, 1)$. But then the Banach lemma shows

$$
\left\|\left(I-\bar{Q}_{n} \mathscr{K}\right)^{-1}\right\| \leqslant 1 /(1-\delta)
$$

As (3.10) holds for the modified collocation solution, (3.32) follows from Theorem 5.

In the case $\alpha^{*}>1$ (Example 2), Theorem 6 requires that the mesh be overgraded, so the modification will not damage the rate of convergence. The next result gives more concrete information when the meshes (3.2) are used.

COROllary 7. Suppose the mesh (3.2) is used with $q>\left(r+r^{\prime}\right) / \alpha^{*}$ and $q \geqslant r+r^{\prime}$.

(a) There is an integer constant $i^{*} \geqslant 0$ such that the modification $i(n)=i^{*}$ will do in Theorem 6.

(b) Suppose constants $a>0$ and $\beta \in\left(0,1-\left(r+r^{\prime}\right) / q \alpha^{*}\right)$ are chosen and $i(n)$ is the nearest integer to an ${ }^{\beta}$. Then $\bar{u}_{n}$ and $\bar{u}_{n}^{*}$ are uniquely defined for all $n$ sufficiently large and (3.32) holds.

Proof. As the mesh is $\left(r+r^{\prime},\left(r+r^{\prime}\right) / q\right)$-graded, Theorem 4 and (3.13) show there is a constant $i^{*}$ such that the modification $i(n)=i^{*}$ satisfies (3.18). By (3.13), this modification is $\left(r+r^{\prime}, \alpha^{*}\right)$-acceptable, which proves (a). The modification in (b) is $\left(r+r^{\prime}, \alpha^{*}\right)$-acceptable by (3.13) and satisfies $i(n) \geqslant i^{*}$ for $n$ sufficiently large. Hence (b) follows from (a).

Corollary 7(a) shows that the modification typically involves only a small proportion of the intervals. In practice, however, modification is usually not required, so we would not advocate the use of the modification in 7(b). More pragmatically, instability will be revealed by poor conditioning of the collocation equations, and this will often be revealed by the algorithm used for their solution. In this case modification can be tried until stability is restored. Theorem 6 shows that this will be successful and will not damage the asymptotic rate of convergence. 
4. Numerical Results. The method described here is a modification of the collocation or product integration methods described in [1] or [23]. Nevertheless, it may be helpful to give a concise algorithmic description. Recall we are solving the integral equation (1.1) with the operators given by (1.2). We will use the meshes (3.2) with the constant modification (described in Corollary $7(\mathrm{a})$ ).

(0) Select integers $r \geqslant 1$ and $i^{*} \geqslant 0$, and numbers $0 \leqslant \xi_{1}<\xi_{2}<\cdots<\xi_{r} \leqslant 1$, and $q \geqslant 1$.

(1) Choose $n \geqslant i^{*}$.

(2) Define the points

$$
\begin{aligned}
& x_{i}^{(n)}=(i / n)^{q}, \quad i=0,1, \ldots, n, \\
& x_{i 1}^{(n)}=x_{i-1}^{(n)}+\frac{1}{2}\left(x_{i}^{(n)}-x_{i-1}^{(n)}\right), \quad i \leqslant i^{*}, \\
& x_{i p}^{(n)}=x_{i-1}^{(n)}+\xi_{p}\left(x_{i}^{(n)}-x_{i-1}^{(n)}\right), \quad i^{*}<i \leqslant n, 1 \leqslant p \leqslant r ;
\end{aligned}
$$

and the index set

$$
Q=\left\{(i, 1): 1 \leqslant i \leqslant i^{*}\right\} \cup\left\{(i, p): i^{*}<i \leqslant n, 1 \leqslant p \leqslant r\right\} .
$$

(3) Let $U=\left(U_{i p}:(i, p) \in Q\right) \in \mathbf{R}^{m}, m=\left(n-i^{*}\right) r+i^{*}$, be the solution of the $m \times m$ system of equations: for all $(i, p) \in Q$

$$
\begin{aligned}
& \sum_{\{\rho:(i, \rho) \in Q\}} U_{i \rho}\left(x_{i p}^{(n)}-x_{i-1}^{(n)}\right)^{\rho-1} \\
& \quad-\sum_{(j, \rho) \in Q} U_{j \rho} \int_{x_{j-1}^{(n)}}^{x_{j}^{(n)}} K\left(\frac{x_{i p}^{(n)}}{\sigma}\right)\left(\sigma-x_{j-1}^{(n)}\right)^{\rho-1} \frac{d \sigma}{\sigma}=f\left(x_{i p}^{(n)}\right) .
\end{aligned}
$$

(4) Then

$$
\bar{u}_{n}^{*}(s):=f(s)+\sum_{(j, \rho) \in Q} U_{j \rho} \int_{x_{j-1}^{(n)}}^{x_{j}^{(n)}} K\left(\frac{s}{\sigma}\right)\left(\sigma-x_{j-1}^{(n)}\right)^{\rho-1} \frac{d \sigma}{\sigma}
$$

is the modified product integration solution.

The theory of the previous section shows $\bar{u}_{n}^{*}$, the modified product integration solution, converges to the true solution $u$ as $n \rightarrow \infty$ provided $i^{*}$ is sufficiently large. The rate of convergence is determined from the choice of $r$ and the $\xi_{p}$ (see (3.6)) and $q$ (see Theorem 6).

The purpose of this section is to present some computations illustrating the results of Section 3. We are solving the equation (see Example 1)

$$
u(s)-\frac{\sin \chi \pi}{\pi} \int_{0}^{1} \frac{s u(\sigma) d \sigma}{s^{2}+\sigma^{2}-2 s \sigma \cos \chi \pi}=f(s), \quad 0 \leqslant s \leqslant 1,
$$

with $\chi=.1$ and where $f$ is found analytically so the solution is $u(s)=s^{1 / 2}$.

In the first computations the basis functions are piecewise linear on the mesh (3.2) and the collocation points are specified by taking $\xi_{1}=0$ and $\xi_{2}=\xi$, where $\xi$ is a parameter that will be varied. As $r^{\prime}=0$, these methods are of no practical value except when $\xi=1$. Nevertheless, they converge when the operator is compact [1]. For (4.1) the discretization error is

$$
\begin{aligned}
\left\|\mathscr{K} Q_{n}^{\prime} u\right\| & \leqslant O\left(1 / n^{q / 2}\right), & & q<4 \\
& \leqslant O\left(1 / n^{2}\right), & & q>4,
\end{aligned}
$$

and we would expect $\left\|u_{n}^{*}-u\right\|$ to converge at these rates. 
We have not computed $\left\|u_{n}^{*}-u\right\|$ but rather

$$
\left|u_{n}^{*}-u\right|=\max _{i, j}\left\{\left|u_{n}^{*}\left(x_{i j}\right)-u\left(x_{i j}\right)\right|\right\}
$$

where $\left\{x_{i j}\right\}$ are the collocation points. As $u_{n}^{*}\left(x_{i j}\right)=u_{n}\left(x_{i j}\right)$ this is relatively cheap. If $u_{n}^{*}(x)$ is needed for $x \notin\left\{x_{i j}\right\}$, the computation will depend on $u_{n}^{*}$ at $\left\{x_{i j}\right\}$, so (4.3) is a fair measure of the error.

Table 1 shows that with $\xi=\frac{1}{2}$ convergence occurs at the rates expected from (4.2), and indeed the eigenvalues of $Q_{n} \mathscr{K}$ are contained in the spectrum of $\mathscr{K}$, i.e., $[0,1-\chi]$. However, as $\xi$ is moved closer to zero, "spurious" eigenvalues appear. At about $\xi=.10222 \ldots$ the principal eigenvalue of $Q_{n} \mathscr{K}, \lambda^{(n)}$, converges to 1 as $n \rightarrow \infty$. The unmodified method then diverges, but convergence is restored by the modification $i(n)=1$ (Table 2 ).

TABLE 1

\begin{tabular}{r|cccc}
\multicolumn{5}{c}{ Values of $\left|u_{n}^{*}-u\right|$ for $\xi=.5}$. \\
$n$ & $q=1$ & $q=2$ & $q=4$ & $q=6$ \\
8 & $5.89(-2)$ & $2.42(-2)$ & $10.6(-3)$ & $13.6(-3)$ \\
16 & $4.24(-2)$ & $1.23(-2)$ & $3.66(-3)$ & $4.86(-3)$ \\
32 & $3.02(-2)$ & $.615(-2)$ & $1.12(-3)$ & $1.46(-3)$ \\
64 & & & $.312(-3)$ & $.395(-3)$
\end{tabular}

TABLE 2

Modified and unmodified solutions when $q=2$ and $\xi=.1022284654$.

\begin{tabular}{r|cc|cc}
$n$ & $\left|u_{n}^{*}-u\right|$ & $\lambda^{(n)}$ & $\left|\bar{u}_{n}^{*}-u\right|$, & $\lambda^{(n)}$ \\
8 & 48. & 1.00240 & .1205 & $.86279 \pm .0228 \mathrm{i}$ \\
16 & 100. & 1.000644 & .0607 & .87433 \\
32 & 206. & 1.000161 & .0304 & .88503 \\
64 & 391. & 1.000043 & &
\end{tabular}

We have also been asked by the referee to compare our collocation method with the Nyström method ([1]) using sinc quadrature ([29]). Thus define $h=a / n^{1 / 2}$, where the parameter $a$ is about $(2 \pi d / \alpha)^{1 / 2}, \alpha=\frac{1}{2}, d=\chi \pi([29,(4.34)])$. Then the quadrature points are

$$
z_{k}=\exp (k h) /(1+\exp (k h)), \quad k=0, \pm 1, \ldots, \pm n,
$$

with weights

$$
w_{k}=h \exp (k h) /(1+\exp (k h))^{2} .
$$

The Nyström solution to (1.1), $u_{n}^{(N)}$, is then defined by

with

$$
\left(I-\mathscr{K}_{n}\right) u_{n}^{(N)}=f \text {, }
$$

$$
\left(\mathscr{K}_{n} v\right)(s):=\sum_{k=-n}^{n} w_{k} K\left(\frac{s}{z_{k}}\right) \frac{1}{z_{k}} v\left(z_{k}\right)
$$


TABLE 3

$\left|u_{n}^{(N)}-u\right|$ for the sinc-Nyström method and various values of $a$.

\begin{tabular}{r|clll} 
& $a=1.25$ & $a=1.5$ & $a=1.75$ & $a=2$ \\
$n=8$ & .0561 & .0493 & .165 & .613 \\
16 & .0346 & .0176 & .0278 & .0606 \\
32 & .0136 & .00638 & .00347 & .00853
\end{tabular}

TABLE 4

\begin{tabular}{r|ccccl}
\multicolumn{5}{c}{$\left|u_{n}^{*}-u\right|$ for collocation at the Gauss points. } \\
$n=8$ & $q=1$ & $q=4$ & $q=6$ & $q=8$ & $q=10$ \\
16 & .0256 & .00561 & .00498 & .00821 & .0137 \\
32 & .0182 & .00141 & .000658 & .000831 & .00145 \\
& .0129 & .000351 & .0000825 & .0000578 & .0000852
\end{tabular}

These methods are simpler to implement than collocation, as $(\mathscr{K} \phi)(s), \phi \in S_{n}$, need not be found analytically. (Although in our example this calculation is not difficult.) Unfortunately, in (4.1) the Nyström method is much less accurate. Table 3 gives the error

$$
\left|u_{n}^{(N)}-u\right|:=\max _{k}\left\{\left|u_{n}^{(N)}\left(z_{k}\right)-u\left(z_{k}\right)\right|\right\} .
$$

Table 4 compares this with the collocation method using piecewise linear functions with collocation at the Gauss points $\left(r=2, \xi_{1}=\frac{1}{2}(1-1 / \sqrt{3}), \xi_{2}=\frac{1}{2}(1+1 / \sqrt{3})\right)$. For a given $n$, the systems of equations to be solved in the two methods are almost the same size.

We conclude that the sinc method is not competitive here. Note that this is the operator appearing when boundary integral methods are used on domains with corners (in this case a re-entrant corner with an exterior angle of $18^{\circ}$ ) which suggests that sinc methods are not the best methods for boundary integral computations. In contrast, piecewise polynomial collocation has been used extensively in the engineering literature, which motivates the theory here.

Mathematics Department

University of Queensland

St. Lucia, Queensland, 4067 Australia

School of Mathematics

University of Bath

Claverton Down, Bath, BA2 7AY United Kingdom

1. K. E. AtKinson, A Survey of Numerical Methods for the Solution of Integral Equations of the Second Kind, SIAM, Philadelphia, Pa., 1976.

2. K. E. AtKInSON \& F. R. DE Hoog, "The numerical solution of Laplace's equation on a wedge," IMA J. Numer. Anal., v. 4, 1984, p. 19.

3. C. DE BooR, "Good approximation by splines with variable knots," in Spline Functions and Approximation Theory (A. Meir and A. Sharma, eds.), Birkhäuser, Basel, 1972, pp. 57-72. 
4. C. DE Boor, A Practical Guide to Splines, Springer-Verlag, New York, 1978.

5. C. A. Brebbia, J. C. F. Telles \& L. C. Wrobel, Boundary Element Techniques, Springer-Verlag, Berlin and New York, 1984.

6. H. G. BURCHARD, "The degree of convergence of piecewise polynomial approximation on optimal meshes," Trans. Amer. Math. Soc., v. 234, 1977, pp. 531-559.

7. G. A. Chandler, Superconvergence of Numerical Solutions to Second Kind Integral Equations, Thesis, Australian National University, 1979.

8. G. A. Chandler, "Galerkin's method for boundary integral equations on polygonal domains," $J$. A ustral. Math. Soc. Ser. B, v. 26, 1984, pp. 1-13.

9. G. A. ChANDler, "Superconvergent approximations to the solution of a boundary integral equation," SIAM. J. Numer. Anal., v. 23, 1986, pp. 1214-1229.

10. G. A. Chandler \& I. G. Graham, Product Integration-Collocation Methods for Non-Compact Integral Operator Equations, Research Report CMA-R41-85, Centre for Mathematical Analysis, Australian National University, Canberra, 1985.

11. M. Costabel \& E. Stephan, "Boundary integral equations for mixed boundary value problems in polygonal domains and Galerkin approximation," in Mathematical Models and Methods in Mechanics, Banach Centre Publications, vol. 15, PWN, Warsaw, 1985, pp. 175-251.

12. M. Costabel, "Boundary integral operators on curved polygons," Ann. Mat. Pura Appl. (4), v. 33, 1983, pp. 305-326.

13. M. L. Dow \& D. Elliotr, "The numerical solution of singular integral equations over $(-1,1)$," SIAM. J. Numer. Anal., v. 16, 1979, pp. 115-134.

14. R. DE VORE \& K. SCHERER, "Variable knot, variable degree spline approximation to $x^{\beta}$," in Quantitative Approximation (R. De Vore and K. Scherer, eds.), Academic Press, New York, 1980, pp. $121-131$.

15. I. G. Graham \& G. A. Chandler, "High order methods for linear functionals of solutions of second kind integral equations," SIAM J. Numer. Anal. (To appear.)

16. M. A. JASWON \& G. I. SYMm, Integral Equation Methods in Potential Theory and Electrostatics, Academic Press, New York, 1977.

17. V. A. KondRAT'EV, "Boundary problems for elliptic equations in domains with conical or angular points," Trans. Moscow Math. Soc., v. 16, 1967, pp. 227-313.

18. M. G. KREIN, Integral Equations on a Half Line with Kernel Depending Upon the Difference of the Arguments, Amer. Math. Soc. Transl., vol. 22, Amer. Math. Soc., Providence, R.I., 1963, pp. 163-288.

19. U. Lamp, T. Schleicher, E. Stephan \& W. L. Wendland, “Galerkin collocation for an improved boundary element method on a plane mixed boundary value problem," Computing, v. 33, 1984, pp. 269-296.

20. W. MCLEAN, Boundary Integral Methods for the Laplace Equation, Thesis, Australian National University, Canberra, 1985.

21. J. R. Rice, "On the degree of convergence of nonlinear spline approximation," in Approximation with Special Emphasis on Spline Functions (I. J. Schoenberg, ed.), Academic Press, New York, 1969.

22. C. SChNEIDER, "Product integration for weakly singular integral equations," Math. Comp., v. 36 , 1981, pp. 207-213.

23. I. H. SloAn, "A review of numerical methods for Fredholm equations of the second kind," in The Application and Numerical Solution of Integral Equations (R. S. Anderssen et al., eds.), Sijthoff and Noordhoff, Groningen, 1980, pp. 51-74.

24. I. H. SloAN \& A. SPEnCE, "Projection methods for integral equations on the half line," IMA $J$. Numer. Anal., v. 6, 1986, pp. 153-172.

25. I. N. SNEDDON \& S. C. DAS, "The stress intensity factor at the tip of an edge crack in an elastic half plane," Internat. J. Engrg. Sci., v. 9, 1971, pp. 25-36.

26. I. N. Sneddon \& M. Lowengrub, Crack Problems in the Classical Theory of Elasticity, Wiley, New York, 1969.

27. M. P. Stallybrass, "A pressurised crack in the form of a cross," Quart. J. Mech. Appl. Math., v. 23,1970 , pp. $35-48$.

28. M. P. Stallybrass, “A crack perpendicular to an elastic half plane," Internat. J. Engrg. Sci., v. 8, 1970, pp. $351-362$.

29. F. STENGER, "Numerical methods based on Whittaker cardinal, or sinc functions," SIAM Rel"., v. 23, 1981, pp. 165-224.

30. W. L. WENDLAND, "On some mathematical aspects of boundary element methods for elliptic problems," in MAFELAP V (J. R. Whiteman, ed.), Academic Press, New York, 1985. 